\title{
ON THE SEMI-INNER PRODUCT IN LOCALLY CONVEX SPACES
}

\author{
SHIH-SEN CHANG \\ YU-QING CHEN \\ Department of Mathematics \\ Sichuan University \\ Chengdu, Sichuan 610064, PEOPLE'S REPUBLIC OF CHINA \\ BYUNG SOO LEE \\ Department of Mathematics \\ Kyungsung University \\ Pusan 608-736, KOREA
}

(Received March 15, 1995 and in revised form June 20, 1995)

ABSTRACT. The purpose of this paper is to introduce the concept of semi-inner products in locally convex spaces and to give some basic properties

KEY WORDS AND PHRASES: Semi-inner product, duality mapping, upper semi-inner product, lower semi-inner product.

1991 AMS SUBJECT CLASSIFICATION CODES: 46C50

\section{INTRODUCTION}

The concept of semi-inner products in real normed spaces was first introduced by G. Lumer [6], but its history can be traced to S Mazur [8]. Recently, the semi-inner product theory has made great progress (cf. $[9,11]$ ) and it plays an important role in the theory of accretive operators and dissipative operators, differential equations, linear and nonlinear semigroups in Banach spaces and Banach space geometry theory (see $[1,2,3,4,5,7]$ ) The purpose of this paper is to introduce the concept of semi-inner products in locally convex spaces and to study their basic properties. As for the applications of our results, we shall give in another paper.

\section{MAIN RESULTS}

In this section, we shall always assume that $E$ is a real locally convex space generated by a family of seminorms $\left\{p_{2}\right\}_{\imath \in I}$, where $I$ is an index set

PROPOSITION 2.1. For each $x \in E, y \in E$ and $i \in I$, the following hold:

(i) $h^{-1}\left(p_{2}(x+h y)-p_{\imath}(x)\right)$ is a nondecreasing function in $h \in(0,+\infty)$ and it is bounded from below,

(ii) $h^{-1}\left(p_{2}(x)-p_{\imath}(x-h y)\right)$ is nonincreasing in $h \in(0,+\infty)$ and bounded from upper,

(iii) $h^{-1}\left(p_{\imath}(x)-p_{\imath}(x-h y)\right) \leq h^{-1}\left(p_{\imath}(x+h y)-p_{\imath}(x)\right)$ for $h \in(0,+\infty)$

PROOF. (i) For any $h_{1}, h_{2} \in(0,+\infty), h_{1}<h_{2}$, since

$$
\begin{aligned}
p_{\imath}\left(x+h_{1} y\right)-p_{\imath}(x) & =p_{i}\left(x+h_{2} \cdot h_{2}^{-1} h_{1} y\right)-p_{\imath}(x) \\
& =p_{\imath}\left(h_{1} h_{2}^{-1}\left(x+h_{2} y\right)+\left(1-h_{1} h_{2}^{-1}\right) x\right)-p_{\imath}(x) \\
& \leq p_{\imath}\left(h_{1} h_{2}^{-1}\left(x+h_{2} y\right)\right)+p_{\imath}\left(\left(1-h_{1} h_{2}^{-1}\right) x\right)-p_{\imath}(x) \\
& =h_{1} h_{2}^{-1} p_{\imath}\left(x+h_{2} y\right)+\left(1-h_{1} h_{2}^{-1}\right) p_{\imath}(x)-p_{\imath}(x) \\
& =h_{2}^{-1} h_{1}\left(p_{\imath}\left(x+h_{2} y\right)-p_{\imath}(x) .\right.
\end{aligned}
$$


Therefore we have $h_{1}^{-1}\left(p_{\imath}\left(x+h_{1} y\right)-p_{\imath}(x)\right) \leq h_{2}^{-1}\left(p_{\imath}\left(x+h_{2} y\right)-p_{\imath}(x)\right)$.

Moreover, it is obvious that $h^{-1}\left(p_{\imath}(x+h y)-p_{\imath}(x)\right) \geq-p_{\imath}(y)$

(ii) By the same way, we can prove that (ii) is true.

(iii) is obvious

Next, we define

$$
\begin{aligned}
& {[x, y]_{2}^{+}=\lim _{h \rightarrow 0^{+}} h^{-1}\left(p_{\imath}(x+h y)-p_{\imath}(x)\right),} \\
& {[x, y]_{2}^{-}=\lim _{h \rightarrow 0^{+}} h^{-1}\left(p_{\imath}(x)-p_{i}(x-h y)\right) .}
\end{aligned}
$$

Now we list some properties of $[x, y]_{2}^{ \pm}$as follows:

PROPOSITION 2.2. (i) $[x, y]_{2}^{-} \leq[x, y]_{2}^{+}$;

(ii) $\left|[x, y]_{2}^{ \pm}\right| \leq p_{2}(y)$,

(iii) $\left|[x, y]_{2}^{ \pm}-[x, z]_{2}^{ \pm}\right| \leq p_{2}(y-z)$;

(iv) $[x, y]_{2}^{+}=-[x,-y]_{2}^{-}=-[-x, y]_{2}^{-}$;

(v) $[s x, r y]_{2}^{ \pm}=s r[x, y]_{2}^{ \pm}, r, s \geq 0$;

(vi) $[x, y+z]_{2}^{+} \leq[x, y]_{2}^{+}+[x, z]_{2}^{+}$and $[x, y+z]_{2}^{-} \geq[x, y]_{2}^{-}+[x, z]_{2}^{-}$;

(vii) $[x, y+z]_{2}^{+} \geq[x, y]_{2}^{+}+[x, z]_{2}^{-}$and $[x, y+z]_{2}^{-} \leq[x, y]_{2}^{-}+[x, y]_{2}^{+}$;

(viii) $[x, y+\alpha x]_{2}^{ \pm}=[x, y]_{2}^{ \pm}+\alpha p_{\imath}(x), \quad \forall \alpha \in \mathbb{R}$;

(ix) $[x, y]_{\imath}^{+}$is upper semi-continuous in $x, y \in E$ and $[x, y]_{\imath}^{-}$is lower semi-continuous in $x, y \in E$;

(x) If $x(t):[a, b] \rightarrow E$ is differentiable in $t \in(a, b)$ in the sense that

$$
\lim _{\Delta t \rightarrow 0} \frac{p_{2}\left(x(t+\Delta t)-x(t)-x^{\prime}(t) \Delta t\right)}{\Delta t}=0 \text { for all } i \in I
$$

and $m_{\imath}(t)=p_{\imath}(x(t))$, then

$$
\begin{gathered}
D^{+} m_{\imath}(t)=\lim _{h \rightarrow 0^{+}} \frac{m_{\imath}(t+h)-m_{\imath}(t)}{h}=\left[x(t), x^{\prime}(t)\right]_{\imath}^{+}, \\
D^{-} m_{\imath}(t)=\lim _{h \rightarrow 0^{+}} \frac{m_{\imath}(t)-m_{i}(t-h)}{h}=\left[x(t), x^{\prime}(t)\right]_{\imath}^{-1}, \quad i \in I .
\end{gathered}
$$

PROOF. (i)-(v) is obvious.

(vi) Since

$$
\begin{aligned}
h^{-1}\left(p_{\imath}(x+h(y+z))-p_{i}(x)\right) & =h^{-1}\left(p_{i}\left(\frac{1}{2}(x+2 h y)+\frac{1}{2}(x+2 h z)\right)-p_{\imath}(x)\right) \\
& \leq \frac{\frac{1}{2}\left(p_{\imath}(x+2 h y)-p_{\imath}(x)\right)}{h}+\frac{\frac{1}{2}\left(p_{\imath}(x+2 h z)-p_{\imath}(x)\right)}{h},
\end{aligned}
$$

we know that $[x, y+z]_{2}^{+} \leq[x, y]_{2}^{+}+[x, z]_{2}^{+}$. On the other hand, since

$$
h^{-1}\left(p_{\imath}(x)-p_{\imath}(x-h(y+z))\right)=h^{-1}\left(p_{i}(x)-p_{\imath}\left(\frac{1}{2}(x-2 h y)+\frac{1}{2}(x-2 h z)\right)\right),
$$

by the same way we can prove that

$$
[x, y+z]_{2}^{-} \geq[x, y]_{2}^{-}+[x, z]_{2}^{-} .
$$

(vii) By (vi) $[x, y]_{2}^{+}=[x, y+z-z]_{2}^{+} \leq[x, y+z]_{i}^{+}+[x,-z]_{2}^{+}$. By (iv), $[x,-z]_{2}^{+}=-[x, z]_{2}^{-}$, and so $[x, y]_{2}^{+}+[x, z]_{2}^{-} \leq[x, y+z]_{2}^{+}$By (vi) and (iv) again, we have $[x, y+z]_{2}^{-} \leq[x, y]_{2}^{-}+[x, z]_{2}^{+}$

(viii) Since $[x, y+\alpha x]_{2}^{+} \leq[x, y]_{2}^{+}+[x, \alpha x]_{2}^{+}=[x, y]_{2}^{+}+\alpha p_{\imath}(x)$, by (vii) we have $[x, y+\alpha x]_{2}^{+} \geq$ $[x, y]_{2}^{+}+[x, \alpha x]_{2}^{-}=[x, y]_{2}^{+}+\alpha p_{i}(x)$, and so $[x, y+\alpha x]_{2}^{+}=[x, y]_{2}^{+}+\alpha p_{2}(x)$

Similarly we can prove that $[x, y+\alpha x]_{2}^{-}=[x, y]_{2}^{-}+\alpha p_{\imath}(x)$.

(ix) Since 


$$
\left[x_{\tau}, y_{\tau}\right]_{2}^{+} \leq \frac{p_{\imath}\left(x_{\tau}+h y_{\tau}\right)-p_{\imath}\left(x_{\tau}\right)}{h}, \quad \forall h>0
$$

if $x_{\tau} \rightarrow x, y_{\tau} \rightarrow y$, we get

$$
\varlimsup_{\tau}\left[x_{\tau}, y_{\tau}\right]_{\imath}^{+} \leq \varlimsup_{\tau} h^{-1}\left(p_{\imath}\left(x_{\tau}+h y_{\tau}\right)-p_{\imath}\left(x_{\tau}\right)\right)=h^{-1}\left(p_{\imath}(x+h y)-p_{\imath}(x)\right),
$$

and so

$$
\varlimsup_{\tau}\left[x_{\tau}, y_{\tau}\right]_{2}^{+} \leq \lim _{h \rightarrow 0} h^{-1}\left(p_{\imath}(x+h x)-p_{\imath}(x)\right)=[x, y]_{2}^{+} .
$$

On the other hand, since $\left[x_{\tau}, y_{\tau}\right]_{2}^{-} \geq h^{-1}\left(p_{\imath}\left(x_{\tau}\right)-p_{\imath}\left(x_{\tau}-h y_{\tau}\right)\right)$, we have

$$
\frac{\lim }{\tau}\left[x_{\tau}, y_{\tau}\right]_{2}^{-} \geq[x, y]_{2}^{-} \text {. }
$$

(x) Since

$$
\begin{aligned}
& \left|h^{-1}\left(m_{\imath}(t+h)-m_{\imath}(t)\right)-h^{-1}\left(p_{\imath}\left(x(t)+h x^{\prime}(t)\right)-p_{i}(x(t))\right)\right| \\
& =\left|h^{-1}\left(p_{\imath}(x(t+h))-p_{\imath}(x(t)+h x(t))\right)\right| \leq h^{-1} p_{\imath}\left(x(t+h)-x(t)-h x^{\prime}(t)\right) \rightarrow 0, \\
& \text { as } h \rightarrow 0^{+}
\end{aligned}
$$

we know that $D^{+} m(t)=\left[x(t), x^{\prime}(t)\right]_{2}^{+}$.

Similarly we can prove that $D^{-} m(t)=\left[x(t), x^{\prime}(t)\right]_{2}^{-}$

Let $E^{*}$ be the dual space of $E$. For each $i \in I$ we define a mapping $j_{\imath}: E \rightarrow 2^{E^{*}}$ by

$$
j_{2}(x)=\left[f_{2} \in E^{*}: f_{2}(x)=p_{2}(x) \text { and }[x, y]_{2}^{-} \leq f_{2}(y) \leq[x, y]_{2}^{+}, \forall y \in E\right\} .
$$

It is obvious that $j_{2}(x)$ is convex Next we prove that $j_{2}(x) \neq \emptyset$ for each $x \in E$ In fact, for any given $y_{0} \in E, y_{0} \neq 0$ we define

$$
f_{2}\left(\alpha y_{0}\right)=\alpha\left[x, y_{0}\right]_{2}^{+} .
$$

(1) If $\alpha \geq 0$, then $f_{2}\left(\alpha y_{0}\right)=\left[x, \alpha y_{0}\right]_{2}^{+}$,

(2) If $\alpha<0$, then

$$
f_{2}\left(\alpha y_{0}\right)=-|\alpha|\left[x, y_{0}\right]_{2}^{+}=-\left[x,|\alpha| y_{0}\right]_{2}^{+}=\left[x,-|\alpha| y_{0}\right]_{2}^{-}=\left[x, \alpha y_{0}\right]_{2}^{-} \leq\left[x, \alpha y_{0}\right]_{2}^{-} .
$$

Hence we have $f_{i}\left(\alpha y_{0}\right) \leq\left[x, \alpha y_{0}\right]_{2}^{+}$for all $\alpha \in \mathbb{R}$. By Proposition 2.2, $[x, y]_{2}^{+}$is a subadditive function of $y \in E$ By Hahn-Banach theorem [10], there exists a linear function $\tilde{f}_{2}: E \rightarrow \mathbb{R}$ such that $\tilde{f}_{\imath}\left(\alpha y_{0}\right)=f_{2}\left(\alpha y_{0}\right)$ for all $\alpha \in \mathbb{R}$ and $-[x,-y]_{z}^{+} \leq \tilde{f}_{z}(y) \leq[x, y]_{\imath}^{+}, \forall y \in E$,

$$
\text { i.e., } \quad[x, y]_{2}^{-} \leq \tilde{f}_{2}(y) \leq[x, y]_{2}^{+}, \quad\left|\tilde{f}_{2}(y)\right| \leq p_{2}(y) .
$$

This implies that $\tilde{f}_{2} \in j_{2}(x)$.

By the above argument and the Banach-Alaoglu theorem (see [10]) we have the following.

PROPOSITION 2.3. For any $x \in E, i \in I, j_{2}(x)$ is a nonempty weak ${ }^{*}$ compact convex subset of $E^{*}$.

PROPOSITION 2.4. $[x, y]_{2}^{+}=\max \left\{f_{\imath}(y), f_{\imath} \in j_{2}(x)\right\}$;

$$
[x, y]_{\imath}^{-}=\min \left\{f_{2}(y): f_{2} \in j_{2}(x)\right\} .
$$

DEFINITION 2.1. For each $i \in I,(x, y)_{\imath}^{+}=p_{\imath}(x) \cdot[x, y]_{2}^{+}$is called the upper semi-inner product with respect to $i \in I .(x, y)_{2}^{-}=p_{z}(x) \bullet[x, y]_{2}^{-}$is called the lower semi-inner product with respect to $i \in I$

DEFINITION 2.2. For any $i \in I$, we define the mapping $J_{2}: E \rightarrow 2^{E^{*}}$ by

$$
J_{\imath}(x)=p_{\imath}(x) \cdot j_{\imath}(x) \text { for all } x \in E,
$$


and it is called the duality mapping with respect to $i \in I$.

The following results can be obtained from Proposition 2 2-2.4 immediately

PROPOSITION 2.5. The semi-inner product defined in Definition 2.1 has the following properties

(i) $(x, y)_{2}^{-} \leq(x, y)_{2}^{+}$,

(ii) $\left|(x, y)_{2}^{ \pm}\right| \leq p_{2}(x) \cdot p_{2}(y)$,

(iii) $\left|(x, y)_{2}^{ \pm}-(x, z)_{2}^{ \pm}\right| \leq p_{2}(x) \cdot p_{2}(y-z)$,

(iv) $(x, y)_{2}^{+}=-(x,-y)_{2}^{-}=-(-x, y)_{2}^{-}$;

(v) $(s x, r y)_{2}^{ \pm}=s r(x, y)_{2}^{ \pm}, r, s \geq 0$;

(vi) $(x, y+z)_{2}^{+} \leq(x, y)_{2}^{+}+(x, z)_{2}^{+}$and $(x, y+z)_{2}^{-} \geq(x, y)_{2}^{-}+(x, z)_{2}^{-}$;

(vii) $(x, y+z)_{i}^{+} \geq(x, y)_{2}^{+}+(x, z)_{2}^{-}$and $(x, y+z)_{2}^{-} \leq(x, y)_{2}^{-}+(x, z)_{2}^{+}$;

(viii) $(x, y+\alpha x)_{2}^{ \pm}=(x, y)_{2}^{ \pm}+\alpha p_{2}^{2}(x), \quad \forall \alpha \in \mathbb{R}$;

(ix) $(x, y)_{2}^{+}$is upper semi-continuous and $(x, y)_{2}^{-}$is lower semi-continuous;

(x) If $x(t):[a, b] \rightarrow E$ is differentiable in $t \in(a, b)$ in the sense that

$$
\lim _{\Delta t \rightarrow 0} \frac{p_{2}\left(x(t+\Delta t)-x(t)-x^{\prime}(t) \cdot \triangle t\right)}{\Delta t}=0, \quad \forall i \in I,
$$

and $m_{\imath}(t)=p_{z}^{2}(x(t))$, then

$$
D^{+} m_{\imath}(t)=2\left(x(t), x^{\prime}(t)\right)_{\imath}^{+} \quad \text { and } \quad D^{-} m_{\imath}(t)=2\left(x(t), x^{\prime}(t)\right)_{\imath}^{-} .
$$

PROPOSITION 2.6. For any $i \in I, x \in E, J_{\imath}(x)$ is nonempty, weak ${ }^{*}$ compact convex, and

$$
\begin{aligned}
& (x, y)_{2}^{+}=\max \left\{f_{2}(y): f_{2} \in J_{2}(x)\right\} \\
& (x, y)_{2}^{-}=\min \left\{f_{2}(y): f_{2} \in J_{2}(x)\right\} .
\end{aligned}
$$

DEFINITION 2.3. Let $\phi: E \rightarrow \mathbb{R}$ be any given convex function The subdifferential of $\phi$ at $x \in E$ (denoted by $\partial \phi(x))$ is defined by

$$
\partial \phi(x)=\left\{f \in E^{*}: \phi(x)-\phi(y) \leq f(x-y) \text { for all } y \in E\right\} .
$$

THEOREM 2.1. Let $\phi_{\imath}(x)=\frac{1}{2} p_{\imath}^{2}(x), x \in E$, then the subdifferential $\partial \phi_{\mathrm{z}}$ is identical to duality mapping $J_{2}$.

PROOF. Let $f \in J_{2}(x)$, then by (2.1) and Definition 2.2 and the fact that $\left|[x, y]_{2}^{+}\right| \leq p_{2}(y)$, we have

$$
f(x-y)=f(x)-f(y) \geq p_{\imath}^{2}(x)-p_{\imath}(x) \cdot p_{\imath}(y) \geq \frac{1}{2}\left(p_{\imath}^{2}(x)-p_{\imath}^{2}(y)\right),
$$

and so, $f \in \partial \phi_{\imath}(x)$

Conversely, if $f \in \partial \phi_{\imath}(x)$, then

$$
p_{\imath}^{2}(x) \leq p_{\imath}^{2}(y)+2 \cdot f(x-y) \text { for all } y \in E .
$$

Replacing $y$ by $x+h y$ in (2.2) we have

$$
p_{\imath}^{2}(x) \leq p_{\imath}^{2}(x+h y)-2 h \cdot f(y) \quad \text { for all } y \in E \text { and } h \in \mathbb{R} .
$$

When $h>0$, we have

$$
\frac{1}{2}\left(p_{i}(x+h y)+p_{i}(x)\right) \cdot \frac{1}{h}\left(p_{\imath}(x+h y)-p_{\imath}(x)\right) \geq f(y), \quad \forall y \in E .
$$

Letting $h \rightarrow 0^{+}$we have

$$
p_{\imath}(x) \cdot[x, y]_{2}^{+} \geq f(y), \quad \forall y \in E .
$$

If $p_{2}(x)=0$, then $f=0$ Therefore $f \in p_{2}(x) j_{2}(x)=J_{2}(x)$, the desired conclusion is proved If $p_{\imath}(x) \neq 0$, for $h<0$, we have 


$$
f(y) \geq \frac{1}{2}\left(p_{\imath}(x+h y)+p_{\imath}(x)\right) \cdot \frac{1}{h}\left(p_{\imath}(x+h y)-p_{\imath}(x)\right), \quad \forall h<0, \quad y \in E .
$$

Letting $h \rightarrow 0^{-}$, we have

$$
f(y) \geq p_{\imath}(x) \cdot[x, y]_{2}^{-} .
$$

By (2.5) and (2.6), we know that $\frac{f}{p_{1}(x)} \in j_{2}(x)$, i e., $f \in p_{\imath}(x) \cdot j_{\imath}(x)=J_{\imath}(x)$

This completes the proof.

DEFINITION 2.4. Let $A: D(A) \subset E \rightarrow 2^{E}$ be a nonlinear multi-valued mapping $A$ is said to be accretive, if

$$
p_{\imath}(x-y) \leq p_{\imath}(x-y+\lambda(u-v))
$$

for all $x, y \in D(A), u \in A(x), v \in A(y), i \in I, \lambda>0$.

THEOREM 2.2. The following conclusions are equivalent:

(i) $A: D(A) \subset E \rightarrow 2^{E}$ is accretive,

(ii) $[x-y, u-v]_{2}^{+} \geq 0$ for all $x, y \in D(A), u \in A x, v \in A y, i \in I$;

(iii) $(x-y, u-v)_{2}^{+} \geq 0$ for all $x, y \in D(A), u \in A x, v \in A y, i \in I$

PROOF. (i) $\Rightarrow$ (ii) Since $\lambda^{-1}\left(p_{\imath}(x-y+\lambda(u-v))-p_{\imath}(x-y)\right) \geq 0$, let $\lambda \rightarrow 0^{+}$we get (i)

(ii) $\Rightarrow$ (iii) is obvious.

(iii) $\Rightarrow$ (ii). Since $(x-y, u-v)_{2}^{+}=p_{\imath}(x-y)[x-y, u-v]_{2}^{+}$.

(a) If $p_{\imath}(x-y)=0$, then $\lambda^{-1}\left(p_{\imath}(x-y+\lambda(u-v))\right) \geq 0$, and so $[x-y, u-v]_{2}^{+} \geq 0$,

(b) If $p_{\imath}(x-y) \neq 0$, then $[x-y, u-v]_{2}^{+} \geq 0$.

(ii) $\Rightarrow$ (i). By Proposition 2.1, $\lambda^{-1}\left(p_{i}(x-y+\lambda(u-v))-p_{\imath}(x-y)\right)$ is nondecreasing in $\lambda \in(0,+\infty)$ and

$$
\lim _{\lambda \rightarrow 0^{+}} \frac{p_{\imath}(x-y+\lambda(u-v))-p_{\imath}(x-y)}{\lambda}-[x-y, u-v]_{\imath}^{+} \geq 0 .
$$

This completes the proof.

THEOREM 2.3. Let $A: D(A) \subset E \rightarrow 2^{E}$ be an accretive mapping and $x:[0,+\infty) \rightarrow E$ be continuous. If the following conditions are satisfied:

(i) there exists $x^{\prime}(t):[0,+\infty) \rightarrow E$ such that

$$
\lim _{\Delta t \rightarrow 0^{+}} \frac{p_{\imath}\left(x(t+\Delta t)-x(t)-x^{\prime}(t) \Delta t\right)}{\Delta t}=0, \quad \forall i \in I
$$

(ii) $x(0)=x_{0} \in D(A)$;

(iii) $x^{\prime}(t) \in-A x(t)$ a.e. $t \in(0,+\infty)$,

then such an $x(t)$ is unique.

PROOF. Suppose the contrary, there exists another $y:[0,+\infty) \rightarrow E$ which is continuous and satisfies conditions (i)-(iii). Let $m_{\imath}(t)=p_{\imath}(x(t)-y(t))$. By $(X)$ in Proposition 2.2, we know that

$$
D^{-} m_{\imath}(t)=\left[x(t)-y(t), x^{\prime}(t)-y^{\prime}(t)\right]_{\imath}^{-} .
$$

Furthermore, there exist $u(t) \in A x(t)$ and $v(t) \in A y(t)$ such that $x^{\prime}(t)=u(t), y^{\prime}(t)=v(t)$ a.e $t \in(0,+\infty)$, hence we have

$$
D^{-} m_{\imath}(t)=[x(t)-y(t),-u(t)+v(t)]_{\imath}^{-} .
$$

It follows from Theorem 2.2 that $D^{-} m_{\imath}(t) \leq 0$, and so

$$
p_{\imath}(x(t)-y(t)) \leq p_{\imath}(x(0)-y(0))=0 \text { for all } i \in I .
$$

This implies that $x(t)=y(t)$ for all $t \in[0,+\infty)$ 
THEOREM 2.4. Let $M \subset E$ be a nonempty convex subset and $x \in E$ be a given point Then the following conditions are equivalent

(i) $p_{2}\left(y_{0}-x\right) \leq p_{\imath}(y-x)$ for all $y \in M$,

(ii) $\left(y_{0}-x, y-y_{0}\right)_{2}^{+} \geq 0$

PROOF. (i) $\Rightarrow$ (ii) Since $p_{\imath}\left(y_{0}-x\right) \leq p_{\imath}(y-x)$ for all $y \in M$, letting $z=y_{0}+(1-\alpha)\left(y-y_{0}\right)$ for any $y \in M, \alpha \in(0,1)$, then $z \in M$ (since $M$ is convex), and so $p_{\imath}\left(y_{0}-x\right) \leq p_{\imath}\left(y_{0}-x+\right.$ $\left.(1-\alpha)\left(y-y_{0}\right)\right), \alpha \in(0,1), y \in M$,

$$
\text { i.e., } \quad \frac{p_{2}\left(\left(y_{0}-x\right)+(1-\alpha)\left(y-y_{0}\right)\right)-p_{\imath}\left(y_{0}-x\right)}{1-\alpha} \geq 0, \quad \forall y \in M, \alpha \in(0,1) .
$$

Letting $\alpha \rightarrow 1-$ we get

$$
\left[y_{0}-x, y-y_{0}\right]_{2}^{+} \geq 0 \text { for all } y \in M .
$$

(ii) $\Rightarrow$ (i) Since $\left[y_{0}-x, y-y_{0}\right]_{2}^{+} \geq 0$, we have

$$
\frac{1}{h}\left(p_{\imath}\left(\left(y_{0}-x\right)+h\left(y-y_{0}\right)\right)-p_{\imath}\left(y_{0}-x\right)\right) \geq 0, \quad \forall h>0,
$$

i e., $p_{\imath}\left(y_{0}-x\right) \leq p_{\imath}\left(y_{0}-x+h\left(y-y_{0}\right)\right), \forall h>0$. Letting $h \rightarrow 1$ we have

$$
p_{2}\left(y_{0}-x\right) \leq p_{2}(y-x) \text { for all } y \in M .
$$

This completes the proof.

ACKNOWLEDGMENT. The first author was supported by the National Natural Science Foundation of China and the third author was supported in part by NON DIRECTED RESEARCH FUND, Korea Research Foundation, 1994.

\section{REFERENCES}

[1] BARBU, V., Nonlinear Semigroups and Differential Equations in Banach Spaces, Nordhoff, 1976.

[2] BEAUZAMY, B., Introduction to Banach Spaces and Their Geometry, North-Holland, 1982

[3] BROWDER, F.E., Nonlinear operators and nonlinear equations of evolutions in Banach spaces, Proc. Symp. Pure Math., 18, 2 (1972).

[4] KATO, T., Nonlinear semigroups and evolution equations, J. Math. Soc Japan, 19 (1967), 508520.

[5] LAKSHMIKANTHAM, V. and LEELA, S., Nonlinear Differential Equations in Abstract Spaces, Pergamon Press, 1981.

[6] LUMER, G., Semi-inner product spaces, Trans. Amer. Math. Soc., 100 (1961), 29-43.

[7] LUMER, G. and PHIILIPS, R.S., Dissipative operators in a Banach space, Pacific J. Math., 11 (1961), 679-698

[8] MAZUR, S., Über knovexe mengen in lineaeren nonmierten Raumen, Studia Math., 4 (1933), 70 84.

[9] REDHEFFER, R.M. and WALTER, W., A differential inequality for the distance function in normed linear spaces, Math. Ann., 211 (1974), 299-314

[10] RUDIN, W., Functional Analysis, McGraw-Hill Book Company, 1973.

[11] TAPIA, R., A characterization of inner product spaces, Proc. Amer. Math. Soc 49 (1973), 564574 


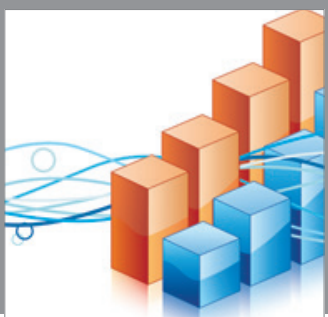

Advances in

Operations Research

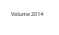

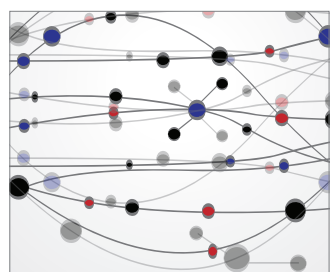

\section{The Scientific} World Journal
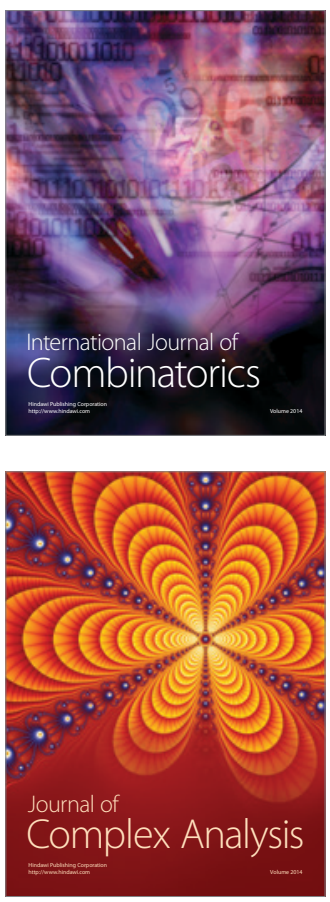

International Journal of

Mathematics and

Mathematical

Sciences
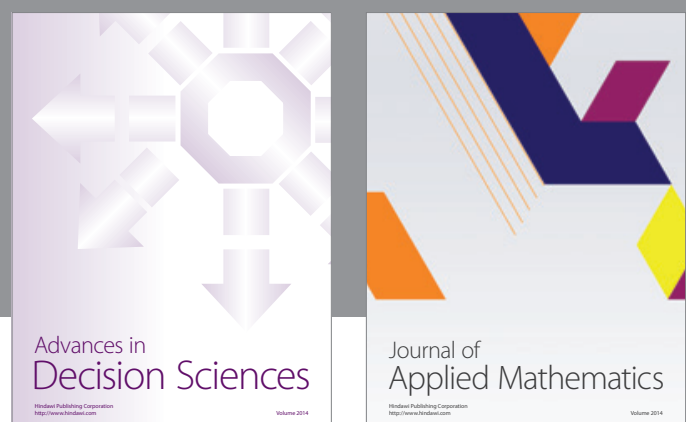

Journal of

Applied Mathematics
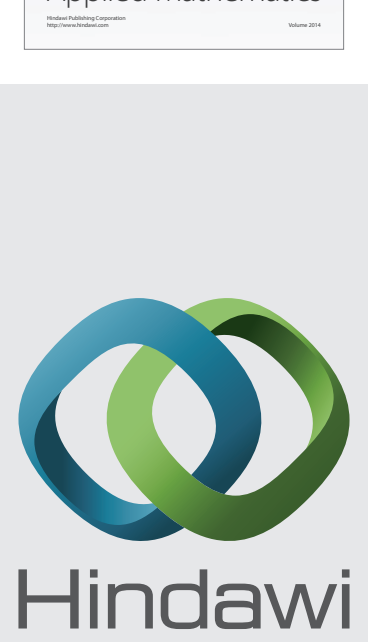

Submit your manuscripts at http://www.hindawi.com
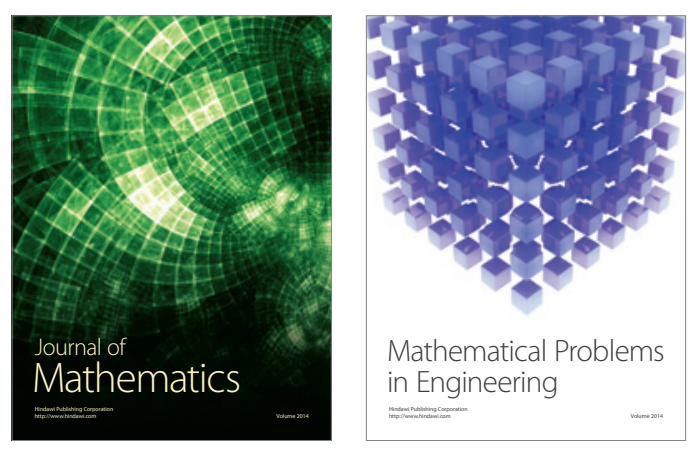

Mathematical Problems in Engineering
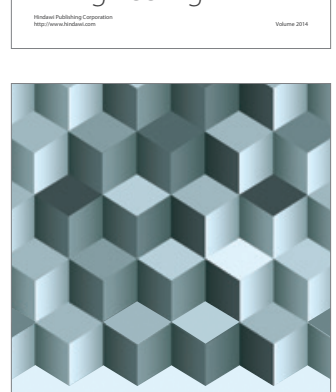

Journal of

Function Spaces
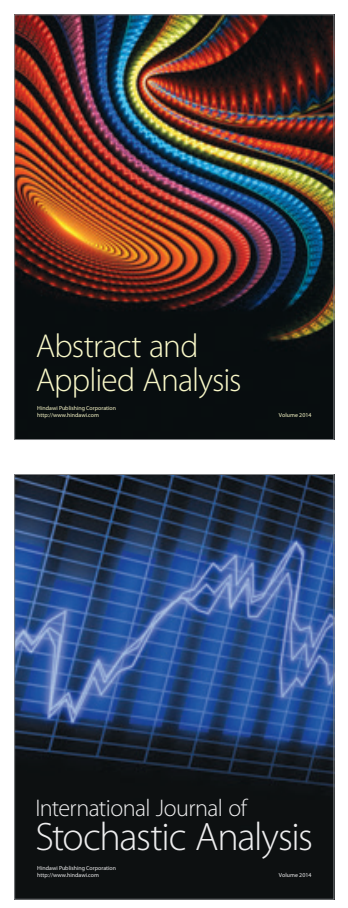

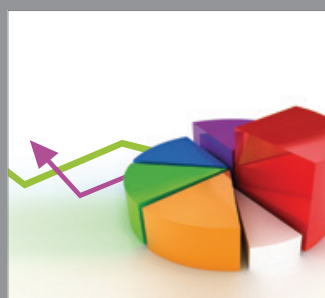

ournal of

Probability and Statistics

Promensencen
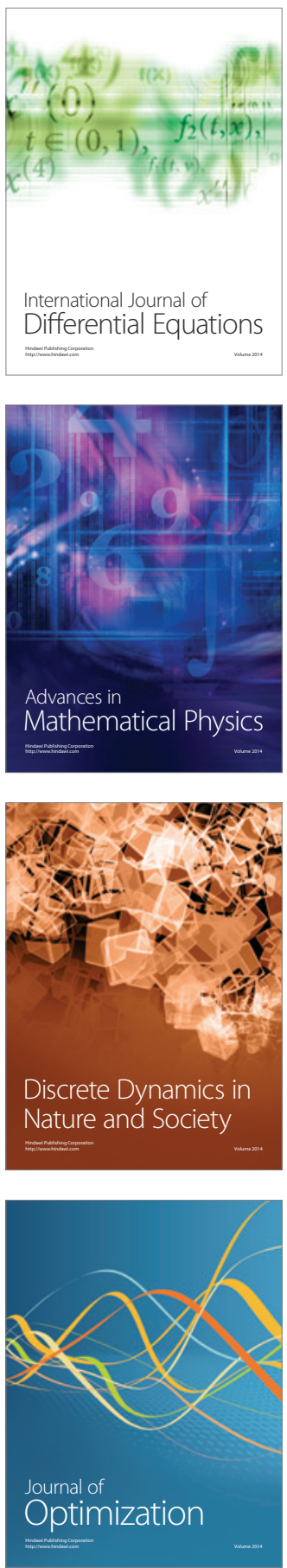